\title{
Effect of cyclic AMP on invertase activity in Neurospora crassa
}

\author{
Hernan Terenzi, $\dagger$ Héctor F. Terenzi and João A. Jorge* \\ Departamento de Biologia, Faculdade de Filosofia, Ciências e Letras de Ribeirão Preto, Universidade de São Paulo, \\ 14049 Ribeirão Preto, São Paulo, Brazil
}

(Received 9 April 1992; revised 17 July 1992; accepted 23 July 1992)

\begin{abstract}
Invertase activity in wild-type and several mutant strains of Neurospora crassa, was significantly enhanced by supplementing the culture medium with cyclic AMP (5.0 mM). Cyclic AMP stimulated invertase by (i) increasing overall enzyme production and (ii) substituting for natural carbohydrate inducers (i.e. galactose or raffinose) as a requirement for maximal enzyme production. The effect of cAMP was specific for the nucleotide and was sensitive to glucose repression. The effect of exogenous cAMP was also studied in mycelial and cell-wall-less derivatives of a 'slime' strain. It was observed that cAMP stimulated invertase production in the mycelial phenotype of 'slime', while its cell-wall-less derivative was totally refractory to the effect of the nucleotide. These findings indicate that the effect of cAMP on $N$. crassa invertase production could be mediated with the participation of cell surface elements.
\end{abstract}

\section{Introduction}

Several lines of evidence indicate the involvement of cyclic AMP (cAMP) in the expression of many phenotypic characteristics of Neurospora crassa and of other filamentous fungi (reviewed by Pall, 1981). A striking example is the $c r-1$ (crisp- 1 ) mutant of $N$. crassa. This mutant grows on solid medium as small, compact colonies, lacking extensive hyphal development and exhibiting dense and precocious conidiation (Perkins, 1959). Biochemical studies demonstrate that the $\mathrm{cr}-1$ mutant exhibits a number of metabolic and enzymic defects, the most prominent being its deficiency in adenylyl cyclase activity (Terenzi et al., 1974, 1976), probably due to a defect of the catalytic protein (Rosenberg \& Pall, 1983).

Supplementation of the culture medium with cAMP cures most, if not all, the phenotypic anomalies of the $\mathrm{cr}-1$ mutant (Terenzi et al., 1976, 1979; Rosenberg \& Pall, 1983), an observation which led Terenzi et al. (1976) to suggest a causal relationship between low cAMP levels and the abnormal phenotype of the mutant. Nevertheless, it is also known that suppressor mutations may partially normalize the $\mathrm{cr}-1$ phenotype without normalizing adenylyl cyclase activity (Terenzi et al., 1979).

* Author for correspondence. Tel. (016) 633 3255; fax (016) 6335015.

† Present address: Instituto e Química da Universidade de São Paulo, Departamento de Bioquimica, Bloco 12 térreo, Cidade Universitária 'Salles de Oliveira', 01498 São Paulo, Brazil.
Hence, it was further suggested that cAMP levels might primarily affect developmental processes and cellular organization in $N$. crassa, and that the observed phenotypic anomalies of $c r-1$ (i.e. overexpression or low activity of a particular enzyme) were probably indirect, rather than direct, consequences of low cAMP levels (Jorge \& Terenzi, 1980; Said \& Terenzi, 1981). Thus, at the present, and despite extensive evidence demonstrating the existence of many elements of a typical cAMP cascade in $N$. crassa, such as cAMP-dependent protein kinase, protein phosphatase, or cAMP phosphodiesterase activities, and some examples of cAMP-dependent protein phosphorylation (Pall, 1981), the primary sites of action of cAMP in this fungus are still poorly understood.

The present work shows the effects of supplementing the culture medium with cAMP on the production of invertase, a $N$. crassa periplasmic enzyme. An earlier study from this laboratory (Terenzi et al., 1979) reported that the $c r-1$ mutant grew poorly in sucrose or raffinose as sources of carbon, and that such nutritional defects were compensated for by the addition of cAMP to the culture medium. At the biochemical level, the failure to assimilate sucrose or raffinose as carbon source could be attributed to a defective activity of invertase. In addition, the observations above seemed to indicate a possible involvement of cAMP on invertase activity. This possibility was confirmed by the present work, not just for the adenylyl cyclase deficient $c r-1$ mutant, but also for other wild-type and mutant strains examined. 


\section{Methods}

Neurospora crassa strains and culture conditions. The following $N$. crassa strains were used throughout the work: FGSC 424 (wild-type); St L. 74A (wild-type); FGSC 488 ( $c r-1$, allele no. B 123); FGSC 2209 (cr-2; al-2; alleles nos. R 2445 and 15300); FGSC 2329 (cr-3; allele no. 2509); FGSC 810 (os- 1 ; allele no. B-135); FGSC 2256 (exo-1; allele no. SF26). All these strains were generously provided by the Fungal Genetics Stock Center (Kansas City, Kansas, USA). We also studied the mycelial intermediate (RCP-3 M) and the stable 'slime' (RCP-3 S) phenotypes obtained from the 'slime'-like segregant RCP-3 $(f z, s g$; $o s-1 ; a l-1)$. Details on the origin and properties of these two strains are given in Pietro et al. (1990).

Mycelial strains were maintained on slants of solid Vogel's (1964) medium supplemented with $2 \%$ (w/v) sucrose (minimal medium). 'Slime' spheroplasts were maintained by weekly transfers on slants of solid Vogel's medium supplemented with $2 \%(\mathrm{w} / \mathrm{v})$ glucose, $0.75 \%$ bactopeptone and $0.75 \%$ yeast extract (complete medium).

Invertase induction experiments were carried out using a two-step culture protocol: the organism was first grown for $24 \mathrm{~h}$ at $30^{\circ} \mathrm{C}$, with orbital agitation ( 140 r.p.m.) in $300 \mathrm{ml}$ Erelenmeyer flasks containing $50 \mathrm{ml}$ minimal liquid medium supplemented with $2 \%(\mathrm{w} / \mathrm{v})$ glucose. The cultures were harvested by filtration, rinsed and resuspended in fresh minimal medium supplemented as described for each experiment. For cultures of 'slime' spheroplasts the medium was also supplemented with $0.5 \mathrm{M}$-sorbitol, and the cells were collected by centrifugation $(500 \mathrm{~g} ; 15 \mathrm{~min})$. The second stage of incubation lasted for $6 \mathrm{~h}$ and the cells were then collected and processed for determination of invertase activity. The culture medium was also saved for invertase determination.

Determination of invertase activity. Mycelial samples were ground in a porcelain mortar with glass beads (diameter 75-150 $\mu \mathrm{m}$ ) and extracted with ten vols of $0.2 \mathrm{M}$-sodium acetate buffer, $\mathrm{pH} 5.0$ (acetate buffer). 'Slime' spheroplasts were disintegrated by vortexing with glass beads for two $90 \mathrm{~s}$ rounds, separated by an interval of cooling in ice. The supernatants of a low speed centrifugation $(500 \mathrm{~g})$ and samples of the culture medium were dialysed overnight against acetate buffer and used directly for invertase determination.

Invertase assay. Invertase activity was assayed as previously described (Pietro et al., 1989). Specific activity (intracellular and extracellular) is expressed as units per mg protein of crude cell extracts. Protein was determined by the Lowry method, using bovine serum albumin (Sigma) as standard.

Cyclic AMP assay. The intracellular concentration of cAMP was measured in mycelia cultured in minimal liquid medium as described in the text. Mycelial samples were collected and extracted in $5 \%(w / v)$ TCA as described by Terenzi et al. (1976). After TCA extraction with ethyl ether, the samples were purified by anionic exchange chromatography using Amprep (SAX) minicolumns (Amersham) according to the supplier's instructions. Cyclic AMP was measured with a cAMP $\left({ }^{3} \mathrm{H}\right)$ assay kit (Amersham) according to the supplier's instructions.

Chemicals. Glucose, galactose, raffinose, sorbitol, cAMP and cGMP were purchased from Sigma. Culture medium reagents were from Difco. All other chemicals were of the best purity available.

\section{Results}

The production of invertase was examined under different physiological conditions: carbon catabolite repression (glucose); induction (galactose or raffinose)
Table 1. Effect of cAMP on invertase production in $N$. crassa strains incubated with varying carbon sources

All strains were first grown for $24 \mathrm{~h}$ at $30^{\circ} \mathrm{C}$, with agitation, in minimal medium supplemented with $2.0 \%(w / v)$ glucose. At that time the cultures were harvested by filtration and resuspended in fresh medium with or without $5.0 \mathrm{mM}$-cAMP and the carbon sources indicated. After $6 \mathrm{~h}$ incubation at $30^{\circ} \mathrm{C}$, the cultures were harvested and invertase activity was determined in the culture medium and cell extracts as in Methods. Intracellular and extracellular specific activities are expressed as units per $\mathrm{mg}$ of crude extracted protein. The results show the average \pm SD of triplicate cultures. Where not indicated, the SD values were lower than $0 \cdot 1$.

\begin{tabular}{|c|c|c|c|c|}
\hline \multirow{3}{*}{$\begin{array}{l}\text { Carbon source } \\
(w / v)\end{array}$} & \multicolumn{4}{|c|}{ Invertase specific activity } \\
\hline & \multicolumn{2}{|c|}{ Cell extract } & \multicolumn{2}{|c|}{ Culture filtrate } \\
\hline & Control & cAMP & Control & cAMP \\
\hline \multicolumn{5}{|c|}{ Wild-type (FGSC 424) } \\
\hline $\begin{array}{l}\text { Glucose } 2.0 \% \\
\text { Galactose } 1.4 \% \\
\text { Raffinose } 2.0 \% \\
\text { None }\end{array}$ & $\begin{array}{l}0.3 \\
9 \cdot 6 \pm 0.7 \\
9 \cdot 6 \pm 0.7 \\
3.5 \pm 0.4\end{array}$ & $\begin{array}{l}0 \cdot 5 \\
14 \cdot 0 \pm 1 \cdot 7 \\
19 \cdot 9 \pm 2 \cdot 4 \\
17 \cdot 4 \pm 2 \cdot 6\end{array}$ & $\begin{array}{r}<0 \cdot 1 \\
0 \cdot 3 \\
0 \cdot 3 \\
0 \cdot 2\end{array}$ & $\begin{array}{r}<0.1 \\
0.3 \\
0.5 \\
0.4\end{array}$ \\
\hline \multicolumn{5}{|c|}{ Wild-type (S.t.L. 74A) } \\
\hline $\begin{array}{l}\text { Glucose } 2.0 \% \\
\text { Galactose } 1 \cdot 4 \% \\
\text { Raffinose } 2.0 \% \\
\text { None }\end{array}$ & $\begin{array}{l}0 \cdot 2 \\
8 \cdot 2 \pm 0.5 \\
9 \cdot 0 \pm 0.8 \\
2 \cdot 9 \pm 0.6\end{array}$ & $\begin{array}{l}0 \cdot 4 \\
12 \cdot 2 \pm 1 \cdot 2 \\
17 \cdot 8 \pm 1 \cdot 2 \\
14 \cdot 4 \pm 1 \cdot 4\end{array}$ & $\begin{array}{r}<0 \cdot 1 \\
0 \cdot 2 \\
0 \cdot 3 \\
0 \cdot 2\end{array}$ & $\begin{array}{r}<0.1 \\
0.3 \\
0.5 \\
0.4\end{array}$ \\
\hline \multicolumn{5}{|l|}{$c r-1$} \\
\hline $\begin{array}{l}\text { Glucose } 2.0 \% \\
\text { Galactose } 1.4 \% \\
\text { Raffinose } 2 \cdot 0 \% \\
\text { None }\end{array}$ & $\begin{array}{l}0.2 \\
1.2 \\
1 \cdot 0 \\
0.9\end{array}$ & $\begin{array}{l}0 \cdot 2 \\
4 \cdot 4 \pm 0.5 \\
3 \cdot 2 \pm 0.4 \\
5 \cdot 3 \pm 0.6\end{array}$ & $\begin{array}{l}<0.1 \\
<0.1 \\
<0.1 \\
<0.1\end{array}$ & $\begin{array}{r}<0.1 \\
0.1 \\
<0.1 \\
<0.1\end{array}$ \\
\hline \multicolumn{5}{|l|}{$o s-1$} \\
\hline $\begin{array}{l}\text { Glucose } 2.0 \% \\
\text { Galactose } 1.4 \% \\
\text { Raffinose } 2.0 \% \\
\text { None }\end{array}$ & $\begin{array}{r}0.5 \\
9 \cdot 8 \pm 0.9 \\
12 \cdot 4 \pm 1 \cdot 2 \\
4.7 \pm 0 \cdot 4\end{array}$ & $\begin{array}{l}0.4 \\
30 \cdot 9 \pm 3 \cdot 7 \\
21 \cdot 4 \pm 1 \cdot 9 \\
35 \cdot 7 \pm 4 \cdot 3\end{array}$ & $\begin{array}{r}<0.1 \\
1.0 \\
1.5 \\
0.9\end{array}$ & $\begin{array}{l}<0 \cdot 1 \\
3 \cdot 0 \pm 0 \cdot 4 \\
2 \cdot 7 \pm 0 \cdot 2 \\
4 \cdot 1 \pm 0.5\end{array}$ \\
\hline \multicolumn{5}{|l|}{ exo-1 } \\
\hline $\begin{array}{l}\text { Glucose } 2.0 \% \\
\text { Galactose } 1.4 \% \\
\text { Raffinose } 2.0 \% \\
\text { None }\end{array}$ & $\begin{array}{l}1 \cdot 0 \\
63 \cdot 0 \pm 9 \cdot 5 \\
34 \cdot 5 \pm 3 \cdot 5 \\
76 \cdot 8 \pm 6.9\end{array}$ & $\begin{array}{r}0.8 \\
96.6 \pm 11.5 \\
81.9 \pm 7.4 \\
208.5 \pm 20.6\end{array}$ & $\begin{array}{l}<0 \cdot 1 \\
1 \cdot 3 \\
1 \cdot 0 \\
5 \cdot 8 \pm 0.6\end{array}$ & $\begin{array}{l}<0 \cdot 1 \\
4 \cdot 4 \\
2 \cdot 4 \\
5 \cdot 7 \pm 0 \cdot 7\end{array}$ \\
\hline
\end{tabular}

and carbon derepression (absence of carbohydrate). The strains studied (Table 1) included a $c r-1$ strain, two other mutant strains exo- 1 and $o s-1$ which are known to exhibit abnormal control of exoenzyme production (Gratzner, 1972; Trevithick \& Metzenberg, 1966), and two wildtype strains. Confirming the initial suspicion, it was found that the activity of invertase in $c r-1$ cultures was very low under all conditions tested, being on average about one-tenth of that of the wild-type. The os-1 mutant produced invertase activity at levels comparable to those of the wild-type, considering the total values, but secreted about three times more enzyme into the culture medium. Invertase synthesis was constitutive for the exo- 1 
mutant, i.e. inducer substrates were not required for maximal enzyme production. In addition, the exo- 1 strain synthesized and secreted into the medium 5-10 times more enzyme than the wild-type. The observed behaviour of the exo-1 mutant accorded with observations by Gratzner \& Sheenan (1969) describing abnormally high secretion of exoenzymes by this strain.

Invertase production was enhanced quite significantly by supplementation of the culture medium with cAMP (Table 1). This was observed, not just for $c r-1$ cultures, but somewhat surprisingly, for all strains tested, including the two wild type strains and the hypersecreting mutant exo-1. The amount of invertase released into the culture fluid was also increased by cAMP supplementation, but to a minor extent in comparison with cell extract activities, such that cAMP-treated cultures retained proportionally more enzyme in the cell mass. On average, the activity of cell-extract invertase, for cAMPtreated cultures, was about two-fold higher than that of controls, either for cultures incubated with glucose or with the inducer substrates, but was even higher (five- to seven-fold) for carbon-derepressed cultures of the inducible strains. The cAMP stimulus to invertase production in carbon derepressed cultures of the constitutive exo-1 strain was lower $(2 \cdot 7$-fold) than that observed for the other inducible strains.

Together, these results suggested that cAMP stimulated invertase synthesis in all the strains tested by: (i) increasing the overall enzyme production and (ii) substituting for the inducer carbohydrates as a requirement for maximal enzyme production. In other words, in the presence of cAMP, invertase synthesis becomes constitutive. Another point of analogy between the effect of cAMP and that of the inducer carbohydrates, on invertase production, was that both were abolished by glucose repression (Table 2).

Under conditions of carbon derepression, invertase synthesis was stimulated by cAMP almost immediately after exposing the cells to the nucleotide (Fig. 1). Enzyme activity increased steadily after a lag of approximately $30 \mathrm{~min}$ and at the end of the experimental period was about five times higher than that of the control cultures. In the absence of cAMP, carbon derepressed cultures accumulated invertase mostly during the first $2-3 \mathrm{~h}$ of incubation (Fig. 1). The production of the enzyme reached a plateau thereafter, as though the biochemical event triggering invertase derepression was no longer active. Interestingly, a brief exposure of the mycelium to exogenous cAMP ( $30 \mathrm{~min}$ ) was sufficient to enhance invertase activity to half of the value observed in cultures incubated with the nucleotide throughout the experimental period.

The stimulatory effect of cAMP on invertase activity was specific for this nucleotide, and was not imitated by

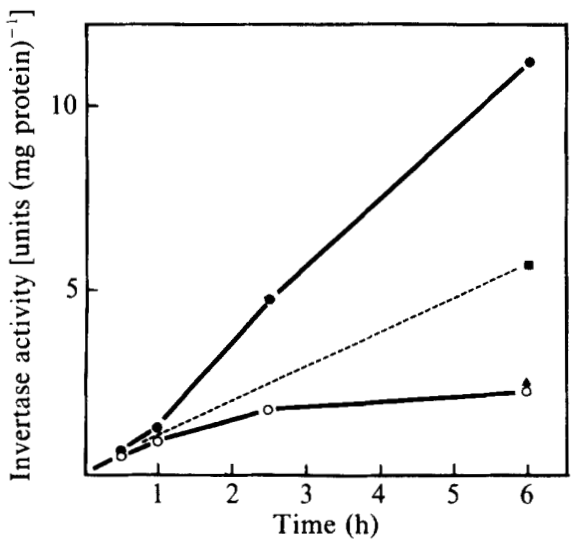

Fig. 1. Time-course of invertase production by carbon-derepressed cultures of the wild-type (FGSC 424) incubated in the presence or absence of cyclic AMP. The organism was grown for $24 \mathrm{~h}$ at $30^{\circ} \mathrm{C}$ with agitation. Mycelium was harvested and resuspended in fresh Vogel's medium without a carbohydrate source, with (closed symbols) or without (O) $5.0 \mathrm{mM}$-cAMP, and incubated as before. At the indicated times, cultures were harvested and invertase activity was determined in the cell extracts. $\Delta$, Culture harvested after $15 \mathrm{~min}$ and resuspended in the same medium but without cAMP; $\square$, culture harvested after 30 min and resuspended in the same medium but without cAMP; culture maintained in medium supplemented with cAMP throughout the experiment. Other details as in Methods.

Table 2. Effect of glucose on the stimulation of invertase synthesis by cAMP

A wild-type strain (FGSC 424) was used. Other details were as described in the legend to Table 1.

\begin{tabular}{|c|c|c|c|c|}
\hline \multirow{3}{*}{$\begin{array}{l}\text { Carbon source } \\
(w / v)\end{array}$} & \multicolumn{4}{|c|}{ Invertase specific activity } \\
\hline & \multicolumn{2}{|c|}{ Cell extract } & \multicolumn{2}{|c|}{ Culture filtrate } \\
\hline & Control & cAMP & Control & cAMP \\
\hline Glucose $2.0 \%$ & $0 \cdot 1$ & $0 \cdot 2$ & $<0 \cdot 1$ & $<0 \cdot 1$ \\
\hline Raffinose $2.0 \%$ & $8.8 \pm 0.8$ & $17 \cdot 2 \pm 1.9$ & $0 \cdot 3$ & 0.5 \\
\hline Raffinose $2.0 \%$ plus & & & & \\
\hline glucose $2.0 \%$ & $0 \cdot 2$ & 0.1 & $<0.1$ & $<0 \cdot 1$ \\
\hline Galactose $2.0 \%$ & $7.8 \pm 0.9$ & $13 \cdot 2 \pm 1 \cdot 6$ & $0 \cdot 4$ & $0 \cdot 6$ \\
\hline $\begin{array}{l}\text { Galactose } 1.4 \% \text { plus } \\
\text { glucose } 2.0 \%\end{array}$ & 0.2 & $0 \cdot 1$ & $<0.1$ & $<0 \cdot 1$ \\
\hline
\end{tabular}

other adenine nucleotides tested (Table 3). On the other hand, some stimulus was also observed in the presence of cyclic GMP, but the maximum invertase activity in the presence of this nucleotide was about half of that obtained with CAMP, and required a two-fold higher concentration. The effect of cAMP on invertase production was concentration-dependent (Fig. 2), and reached a maximum at $5.0 \mathrm{~mm}$, either in the presence or absence of inducer carbohydrates. 


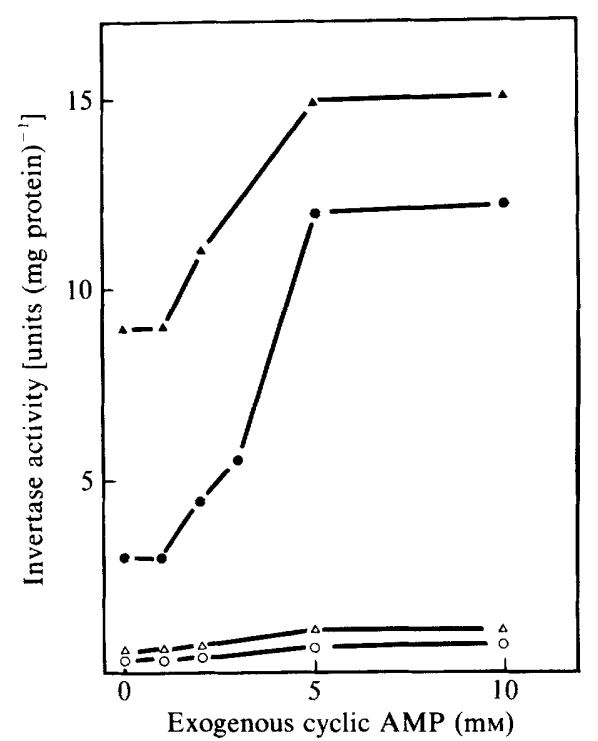

Fig. 2. Dose effect of exogenous cyclic AMP on invertase production by carbon-derepressed, or induced (galactose), wild-type (FGSC 424) cultures. Experimental conditions as Fig. 1. After the first stage of growth $(24 \mathrm{~h})$, cultures were harvested and resuspended in fresh Vogel's medium supplemented with $1.4 \%$ galactose $(\triangle, \Delta)$, or without a carbohydrate source $(O, O)$, and cyclic AMP as indicated. After $6 \mathrm{~h}$ incubation at $30^{\circ} \mathrm{C}$, cultures were harvested and invertase activity was determined in cell extracts (filled symbols) and culture filtrates (open symbols). Other details as in Methods.

Table 3. Effect of several nucleotides on the invertase activity of carbon-derepressed cultures

A wild-type strain (FGSC 424) was used. After the first stage of growth $(24 \mathrm{~h})$ the cultures were resuspended in minimal medium lacking a carbohydrate source, and with the indicated supplements. Other details were as described in the legend to Table 1.

\begin{tabular}{lcc}
\hline \hline \multirow{2}{*}{$\begin{array}{c}\text { Nucleotide } \\
(10 \mathrm{mM})\end{array}$} & \multicolumn{2}{c}{ Invertase specific activity } \\
\cline { 2 - 3 } & Cell extract & Culture filtrate \\
\hline cAMP & $12.0 \pm 1.8$ & 0.5 \\
ADP & 1.8 & $0 \cdot 2$ \\
AMP & $2 \cdot 2 \pm 0.2$ & 0.2 \\
ATP & 1.3 & $0 \cdot 1$ \\
cGMP (20 mM) & $6.4 \pm 0.9$ & 0.4 \\
No additions & $2 \cdot 1 \pm 0.2$ & 0.2 \\
\hline \hline
\end{tabular}

An important point to be made concerns the specificity of the cAMP effect in the adenylyl cyclase deficient $c r-1$ mutant. It was seen (Table 1) that cAMP stimulated invertase production in this strain, but not to a higher proportion than for the other strains tested. Hence, even in the presence of CAMP, the production of invertase by $c r-l$ was still defective compared to the wild-type. Under our experimental conditions culture growth was not extensive, and therefore there was little chance for
Table 4. Invertase production and effect of cyclic AMP on carbon-derepressed cultures of wild-type, three 'crisp' mutants and a 'crisp' mutant carrying a suppressor mutation

After the first stage of growth $(24 \mathrm{~h})$ the cultures were resuspended in minimal medium lacking a carbohydrate source and supplemented or not with 5.0 mM-cAMP. Other details were as described in the legend to Table 1.

\begin{tabular}{|c|c|c|c|c|}
\hline \multirow[b]{3}{*}{ Strain } & \multicolumn{4}{|c|}{ Intertase specific activity } \\
\hline & \multicolumn{2}{|c|}{ Cell extract } & \multicolumn{2}{|c|}{ Culture filtrate } \\
\hline & Control & cAMP & Control & cAMP \\
\hline Wild-type (FGSC 424) & $3 \cdot 2 \pm 0 \cdot 2$ & $11.6 \pm 1.9$ & $0 \cdot 2$ & $0 \cdot 4$ \\
\hline$c r-1$ & 0.9 & $4.8+0.5$ & $<0 \cdot 1$ & $0 \cdot 2$ \\
\hline$c r-2$ & $14 \cdot 2 \pm 1 \cdot 3$ & $38.2 \pm 3.8$ & 1.9 & $6.2 \pm 0.6$ \\
\hline$c r-3$ & $9.2 \pm 0.4$ & $41 \cdot 2 \pm 4.9$ & $1 \cdot 0$ & $6.4 \pm 0.8$ \\
\hline$(s u)-c r-1$ & $18.8 \pm 1.7$ & $96.9 \pm 13.6$ & $0 \cdot 6$ & $4.0 \pm 0.4$ \\
\hline
\end{tabular}

cAMP to induce reversion of the $c r-1$ morphology. Thus, it may be that the defective invertase production in this strain was principally a consequence of its abnormal morphology. This idea was strengthened by the observations shown in Table 4, demonstrating that a $\mathrm{cr}$ - 1 strain carrying a suppressor mutation, $(s u) c r-1$, produced and secreted even higher levels of invertase than the wildtype. Significantly, early studies showed that the (su) $\mathrm{cr}-1$ mutation cures most of the phenotypic defects of $c r-1$, except the defective activity of adenylyl cyclase (Terenzi et al., 1979). On the other hand, other mutant strains exhibiting a crisp-like morphology, namely $c r-2$ and $c r-3$ which have normal cAMP levels (Terenzi et al., 1976), produced rather high levels of invertase activity (Table 4). This, it seemed likely that the invertase defect in $c r-1$ was one of a number of enzymic alterations secondary to the adenylyl cyclase deficiency exhibited by the mutant (Jorge \& Terenzi, 1980; Said \& Terenzi, 1981; Savtchenko et al., 1986).

As stated above, invertase was produced constitutively in cAMP-supplemented cultures, in the absence of a carbohydrate source (see also Table 1). This observation could be taken as an indication that the induction of invertase by galactose or other appropriate carbohydrate was associated with increased levels of endogenous cAMP. This hypothesis was tested using a wild-type strain in the experiments represented in Fig. 3. The levels of cAMP in mycelia resuspended in glucose, galactose or in the absence of carbohydrate, changed little throughout the $2 \mathrm{~h}$ experimental period (Fig. $3 b$ ). During this time, invertase synthesis was very active in the cultures supplemented with galactose (data not shown). By sampling the cultures at shorter times after resuspension in the new media, transient, quite modest peaks of cAMP were detected (Fig. $3 a$ ). Nevertheless, the highest 


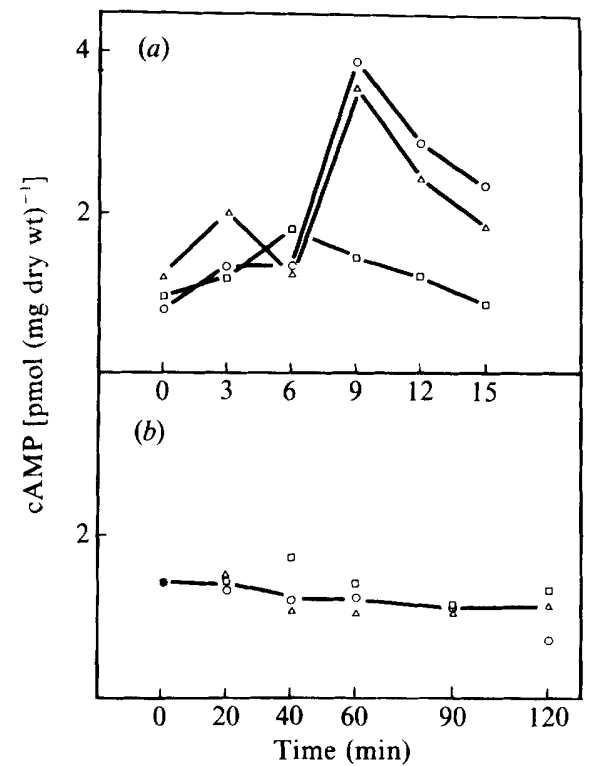

Fig. 3. Cyclic AMP levels upon resuspension of glucose-grown mycelium (wild-type FGSC 424) in fresh medium supplemented with glucose or galactose, or without a carbohydrate source. The organism was grown for $24 \mathrm{~h}$ at $30^{\circ} \mathrm{C}$ with agitation, in Vogel's medium supplemented with $2.0 \%(\mathrm{w} / \mathrm{v})$ glucose. Mycelium was harvested and resuspended in fresh Vogel's medium supplemented with $0,2.0 \%$ (w/v) glucose: $\square, 1.4 \%(w / v)$ galactose or $\triangle$, without a carbohydrate source. At the times indicated, mycelium samples were withdrawn and intracellular cAMP levels determined as described in Methods. (a) Short time-scale experiment; (b) longer time-scale.

relative increase was that of the culture supplemented with glucose, an observation which seemed to rule out a possible relationship of the cAMP with invertase induction. The transient changes observed in intracellular levels of cAMP probably reflected mechanical stress from the experimental manipulation of the mycelia, as was earlier observed in $N$. crassa by Pall (1977).

Although the previous results argued against large increases in endogenous cAMP during invertase induction, it must be stressed that these experiments would not detect a change, even if significant, occurring in a cell compartment which somehow might influence invertase activity. $N$. crassa invertase is a periplasmic enzyme (Metzenberg, 1963) and it is well supported by biochemical and genetic evidence that mutations which affect the structure and composition of the cell wall commonly affect the production of exoenzymes, including invertase. This may be the case for the os- 1 and exo- 1 mutant strains, in which the present results demonstrated misregulation of invertase (see Table 1). An extreme example of this phenomenon is described in studies from our laboratory, about the anomalous pattern of control of invertase and other carbon-regulated exoenzymes in cellwall-deficient phenotypes of the $f z$; sg; os-1 ('slime') triple mutant of $N$. crass $a$. These studies demonstrate, by
Table 5. Effect of cyclic AMP on invertase production in cultures of the mycelial $(M)$ and wall-less $(S)$ phenotypes obtained from the 'slime'-like segregant RCP-3

The strains were grown for $24 \mathrm{~h}$ at $30^{\circ} \mathrm{C}$ with agitation, in complete medium supplemented with $2.0 \%(\mathrm{w} / \mathrm{v})$ glucose. Cultures were harvested by centrifugation (stable 'slime') or filtration (mycelial intermediate) and resuspended in fresh minimal medium with or without $5.0 \mathrm{mM}-\mathrm{cAMP}$ and the carbon sources indicated. After $6 \mathrm{~h}$ incubation at $30^{\circ} \mathrm{C}$, the cultures were harvested and invertase activity was determined in the culture filtrates and cell extracts as described in Methods. Other details were as in the legend to Table 1 .

\begin{tabular}{|c|c|c|c|c|}
\hline \multirow{3}{*}{$\begin{array}{c}\text { Carbon source } \\
(w / v)\end{array}$} & \multicolumn{4}{|c|}{ Invertase specific activity } \\
\hline & \multicolumn{2}{|c|}{ Cell extract } & \multicolumn{2}{|c|}{ Culture filtrate } \\
\hline & Control & cAMP & Control & cAMP \\
\hline \multicolumn{5}{|l|}{$R C P-3 M$} \\
\hline Glucose $\quad 2 \cdot 0 \%$ & $0 \cdot 1$ & $2 \cdot 6$ & $0 \cdot 2$ & $0 \cdot 2$ \\
\hline Galactose $1.4 \%$ & $39 \cdot 3 \pm 4 \cdot 7$ & $67 \cdot 1 \pm 8 \cdot 0$ & $5 \cdot 1 \pm 0.4$ & $5.6 \pm 0.4$ \\
\hline Raffinose $2.0 \%$ & $5.7 \pm 0.6$ & $30.6 \pm 3.8$ & $\overline{1 \cdot 3}$ & $11 \cdot 1 \pm 1 \cdot 3$ \\
\hline None & $12 \cdot 5 \pm 1 \cdot 3$ & $69.6 \pm 4.0$ & $2 \cdot 5 \pm 0.3$ & $3.5 \pm 0.3$ \\
\hline \multicolumn{5}{|l|}{$R C P-3 S$} \\
\hline Glucose $\quad 2.0 \%$ & $1.9 \pm 0.2$ & $1.6 \pm 0.2$ & $4 \cdot 0 \pm 0.2$ & $4.0 \pm 0.3$ \\
\hline Galactose $1.4 \%$ & $2.6 \pm 0.3$ & $3.0 \pm 0.4$ & $3.0 \pm 0.2$ & $3.8+0.2$ \\
\hline Raffinose $2.0 \%$ & $2.7 \pm 0.2$ & $3 \cdot 0 \pm 0.2$ & $4 \cdot 8 \pm 0.5$ & $4.8 \pm 0.4$ \\
\hline None & $2.6 \pm 0.4$ & $2 \cdot 2 \pm 0 \cdot 2$ & $9 \cdot 6 \pm 1.0$ & $9.1 \pm 0.8$ \\
\hline
\end{tabular}

comparing the behaviour of cell-wall-less and cell-wallforming phenotypes, obtained from a single 'slime' ascospore isolate, that the irreversible loss of the cell wall has drastic consequences on the control mechanisms of invertase synthesis. Specifically, 'slime' spheroplasts became insensitive to carbon catabolite repression, and to substrate-mediated induction of exoenzymes, while the cell-wall-forming phenotype of the same strain behaves normally (Pietro et al. 1989, 1990; Polizeli et al., 1990).

In view of these precedents, it seemed worthwhile to examine the effect of cAMP on the production of invertase by the mycelial and wall-less phenotypes of the 'slime' strain RCP-3 (Pietro et al., 1990). From these results, shown in Table 5, it seemed evident that the stimulatory effect of cAMP on invertase synthesis was processed with the participation of cell surface elements. Indeed, the mycelium-forming phenotype of strain RCP-3 was fully responsive to galactose induction and to glucose repression of invertase, and also to the stimulatory effect of cAMP as observed for other inducible mycelial strains (Table 1). In contrast, the stable 'slime', derived from the mycelial form of strain RCP-3, was refractory to all these exogenous agents, producing invertase constitutively. It is worth noting that the specific production of invertase (cells plus medium) in 
cultures of RCP-3 spheroplasts was seven times lower than that of the mycelial phenotype in galactose, but it was twenty-fold higher under conditions of glucose repression. Analogous behaviour was observed by comparing the mycelial and cell-wall-less phenotypes of two other 'slime' strains (data not shown). These data are consistent with previous results (Pietro et al., 1989, 1990) demonstrating that the loss of cell wall in 'slime' seems to turn off many responses of these cells to external stimuli.

\section{Discussion}

The results reported here suggest the involvement of cAMP in the expression of invertase activity in N. crassa. The stimulatory effect of cAMP on invertase synthesis, in carbon-starved mycelium, substituted for that of galactose or raffinose, the carbohydrate inducers. Likewise, the stimulatory effect of cAMP was nullified by carbon catabolite repression. Thus, it is possible that the mechanism of action of exogenous cAMP was of physiological significance.

The biochemical basis of the effects of exogenous cAMP in $N$. crassa is not fully understood, but on the basis of indirect evidence, most studies implicitly suggest that the nucleotide permeates into the cells and acts on intracellular targets, i.e. cAMP-dependent protein kinases (Pall, 1981). Although this hypothetical mechanism of action of exogenous cAMP has not been experimentally confirmed, it seems difficult to justify an alternative mechanism, such as a direct interaction of cAMP with a cell surface receptor. Thus, if we admit as true the involvement of a cAMP cascade in the processes leading to invertase induction, our results suggest the participation of other element(s) downstream of the cAMP site, with an important role in the control of invertase synthesis. Moreover, our results also suggest that a critical element in the cAMP effect was functionally dependent on the integrity of the cell wall. These conclusions rely on the following arguments. First, cAMP supplementation affects the activity of extracellular $N$. crassa enzymes other than invertase, such as NAD phosphohydrolase (Jorge \& Terenzi, 1980), aryl- $\beta$ glucosidase (Said \& Terenzi, 1981) and alkaline protease (Savtchenko et al., 1986). Secondly, most morphologic and metabolic defects in $c r-1$, including that of invertase production, are normalized by suppressor mutations which, nevertheless, do not restore normal adenylyl cyclase activity. Thirdly, the effect of exogenous cAMP on invertase was not observed in the cell-wall-less phenotype of 'slime', but it was observed in the mycelium-forming one.

Up to now, few reports have described the metabolic effects of cAMP in N. crassa. Dumbrava \& Pall (1987) demonstrated that the cAMP deficiency in $c r-1$ is associated with an elevated pool of erythroascorbic acid and UDP-glucuronic acid, and that both pools decline in response to exogenous cAMP. It is known that UDPglucuronic acid is a donor of glucuronate for polysaccharide synthesis, and that Fraction 1 of $N$. crassa cell wall contains glucuronic acid (Mahadevan \& Tatum, 1965). According to Cardemil \& Pincheira (1979) uronic acids may play a significant role in $N$. crassa morphology and growth.

On this basis, it may be reasonable to suggest, as a working hypothesis, that exogenous cAMP supplementation may produce a metabolic effect on cell wall polysaccharide synthesis. As a consequence, the properties of the cell wall and of cell-wall-related proteins would be also affected by cAMP supplementation. This tentative explanation might satisfy for the time being the questions concerning the effects of cAMP on invertase activity raised by the present study. Confirmation of the possible effects of cAMP at the level of cell wall metabolism must await further studies.

This work was supported by grants from Financiadora de Estudos e Projetos, Fundação de Amparo a Pesquisa do Estado de São Paulo and Conselho de Desenvolvimento Científico e Tecnológico (CNPq). H.T. received a Graduate (MASTER) Fellowship from CNPq. J. A. J. and H.F.T. are Research Fellows of CNPq. This work is part of a Dissertation submitted by H.T. to the Department of Biochemistry of the School of Medicine of Ribeirão Preto (University of São Paulo) in partial fulfillment for the requirements for the Master Degree. We thank Carlos de Carvalho Almada and Maurício de Oliveira for technical assistance.

\section{References}

Cardemil, L. \& Pincheira, G. (1979). Characterization of the carbohydrate component of fraction I in the Neurospora crassa cell wall. Journal of Bacteriology 137, 1067-1072.

Dumbrava, V.-A. \& Pall, M. L. (1987). Control of nucleotide and erythroascorbic acid pool by cyclic AMP in Neurospora crassa. Biochimica et Biophysica Acta 926, 331-338.

GratzNer, H. G. (1972). Cell wall alterations associated with the hyperproduction of extracellular enzymes in Neurospora crassa. Journal of Bacteriology 111, 443-446.

Gratzner, H. G. \& Sheenan, D. N. (1969). Neurospora mutant exhibiting hyperproduction of amylase and invertase. Journal of Bacteriology 97, 544-549.

Jorge, J. A. \& TerenzI, H. F. (1980). An enzymatic alteration secondary to adenylyl cyclase deficiency in the $\mathrm{cr}-\mathrm{l}$ (crisp) mutant of Neurospora crassa. Developmental Biology 74, 164-177.

Mahadevan, P. R. \& Tatum, E. L. (1965). Relationship of the major constituents of the Neurospora crassa cell wall to wild type and colonial morphology. Journal of Bacteriology 90, 1073-1081

MetzenberG, R. L. (1963). The localization of $\beta$-fructofuranosidase in Neurospora. Biochimica et Biophysica Acta 77, 455-465.

PaLL, M. L. (1977). Cyclic AMP and the plasma membrane potential in Neurospora crassa. Journal of Biological Chemistry 252, 7146-7150.

PALL, M. L. (1981). Adenosine 3',5'-phosphate in fungi. Microbiological Reviews 45, 462-480.

Perkins, D. D. (1959). New markers and multiple point linkage data in Neurospora. Genetics 44, 1185-1208. 
Pietro, R. C. L. R., Jorge, J. A. \& Terenzi, H. F. (1989). Pleiotropic deficiency in the control of carbon-regulated catabolic enzymes in the 'slime' variant of Neurospora crassa. Journal of General Microbiology 135, 1375-1382.

Pietro, R. C. L. R., Jorge, J. A. \& Terenzi, H. F. (1990). Effects of cell wall deficiency in 'slime' strains of Neurospora crassa: a study on mycelial and wall-less phenotypic derivatives of a single $f z ; s g ; o s-1$ ('slime-like') segregant. Journal of General Microbiology 136, 121 129.

Polizeli, M. L. T. M., Pietro, R. C. L. R., Jorge, J. A. \& Terenzi, H. F. (1990). Effects of cell wall deficiency on the synthesis of polysaccharide-degrading exoenzymes. A study on mycelial and wall-less phenotypes of the $f z, s g, o s-1$ ('slime') triple mutant of Neurospora crassa. Journal of General Microbiology 136, 1463-1468.

Rosenberg, G. B. \& PALL, M. L. (1983). Reconstitution of adenylate cyclase in Neurospora crassa from two components of the enzyme. Archives of Biochemistry and Biophysics 221, 254-260.

SAID, S. \& TERENZI, H. F. (1981). Enzymatic alterations secondary to adenyl cyclase deficiency in the $c r-1$ (crisp) mutant of Neurospora crassa: anomalies of the $\beta$-glucosidase system. Brazilian Journal of Medical and Biological Research 14, 3-10.
Savtchenko, E. S., Terenzi, H. F. \& Jorge, J. A. (1986). Alkaline protease deficiency in the $c r-l$ (crisp) mutant of Neurospora crassa. Brazilian Journal of Medical and Biological Research 19, 27-32.

TERenZI, H. F., Flawia, M. M. \& ToRRes, H. N. (1974). A Neurospora crassa morphological mutant showing reduced adenylate cyclase activity. Biochemical and Biophysical Research Communications 58, 990-996.

Terenzi, H. F., Flawia, M. M., Telles-Iñon, M. T. \& Torres, H. N. (1976). Control of Neurospora crassa morphology by cyclic adenosine 3'5'-monophosphate and dibutyryl cyclic adenosine 3'5'-monophosphate. Journal of Bacteriology 126, 91 99.

Terenzi, H. F., Jorge, J. A., Roselino, J. E. \& Migliorini, R. H. (1979). Adenylyl cyclase deficient $c r-1$ (crisp) mutant of Neurospora crassa: cyclic AMP-dependent nutritional deficiencies. Archives of Microbiology 123, 251-258.

Trevithick, J. R. \& Metzenberg, R. L. (1966). Molecular sieving by Neurospora cell walls during secretion of invertase isozymes. Journal of Bacteriology 92, 1010-1015.

VoGEL, H. J. (1964). Distribution of lysine pathways among fungi: evolutionary implications. American Naturalist 98, 435-446. 